\title{
Determination of zinc in rice grains using DTZ staining and ImageJ software
}

\author{
Rômulo F. Duarte a, b, *, Chanakan Prom-u-thai a, c, **, Douglas C. Amaral b, \\ Valdemar Faquin $^{\text {b }}$, Luiz R.G. Guilherme ${ }^{\text {b }}$, André R. Reis ${ }^{d}$, Eduardo Alves ${ }^{\text {e }}$ \\ a Agronomy Division, Department of Plant Science and Natural Resources, Faculty of Agriculture, Chiang Mai University, Chiang Mai, 50200, Thailand \\ b Department of Soil Science, Federal University of Lavras, P.O. Box 3037, Lavras, MG 37200-000, Brazil \\ ${ }^{\mathrm{c}}$ Lanna Rice Research Center, Chiang Mai University, Chiang Mai, 50200, Thailand \\ d Biosystems Engineering, Faculty of Science and Engineering, Sao Paulo State University, Tupã, SP 17602-496, Brazil \\ e Department of Plant Pathology, Federal University of Lavras, P.O. Box 3037, Lavras, MG 37200-000, Brazil
}

\section{A R T I C L E I N F O}

\section{Article history:}

Received 14 June 2015

Received in revised form 1 October 2015

Accepted 11 November 2015

Available online 1 December 2015

\section{Keywords:}

Oryza sativa L.

Zinc deficiency

Staining intensity

Biofortification

\begin{abstract}
A B S T R A C T
Dithizone (DTZ) staining is a rapid, simple, and inexpensive method that allows the histochemical localization of $\mathrm{Zn}$. In this study, we evaluated genotypic variation in $\mathrm{Zn}$ concentration in brown rice grown in the field using atomic absorption spectrophotometer (AAS) as DTZ staining quantified using Image software. We used dehusked grains from upland rice accessions widely cultivated in Brazil. The DTZ staining showed that the concentration of $\mathrm{Zn}$ varied within and between rice grains. The staining intensity index $(Y)$ provided differences in localization of $\mathrm{Zn}$ across the grain regions. $\mathrm{Zn}$ concentration in brown rice varied in multiple regression analysis, showing major differences in index weighted by stained area of each region (YAW) for the embryo, endosperm, and aleurone, especially in the endosperm and aleurone regions due to a large portion of the area in the kernel. The total YAW among rice accessions was positively correlated with $\mathrm{Zn}$ concentration in grains by chemical analysis. The DTZ staining associated with image software is a promising to estimate $\mathrm{Zn}$ concentration in different grain tissues. Thus, this method may be useful for rapid screening of rice germplasms.
\end{abstract}

() 2015 Elsevier Ltd. All rights reserved.

\section{Introduction}

Zinc $(\mathrm{Zn})$ is an essential micronutrients required for normal and healthy growth of plants, animals, and humans (Alloway, 2008). Zinc deficiency occurs in about half the world's population, and causes general problems associated with growth and development including birth defects in pregnant women, stunted growth of children, and increased susceptibility to infectious diseases (Cakmak, 2008; Graham et al., 2012; Prasad, 2012). The consumption of agricultural products with inadequate amounts of $\mathrm{Zn}$ levels is considered to be the leading primary reason for $\mathrm{Zn}$ deficiency in

\footnotetext{
* Corresponding author. Department of Soil Science, Federal University of Lavras, P.O. Box 3037, Lavras, MG 37200-000, Brazil.

** Corresponding author. Department of Plant Science and Natural Resources, Faculty of Agriculture, Chiang Mai University, Chiang Mai, 50200, Thailand.

E-mail addresses: rfduarte83@gmail.com (R.F. Duarte), chanakan15@hotmail. com (C. Prom-u-thai).
}

humans, especially in developing countries (Alloway, 2008; Pfeiffer and McClafferty, 2007; White and Broadley, 2009; Sharma et al., 2013). Therefore, increasing $\mathrm{Zn}$ concentration in crops such as rice, an important source of energy for more than three billion people living in Asia, could provide a strategy for decreasing the incidence of $\mathrm{Zn}$ deficiency in regions where rice is the staple crop (Bouis and Welch, 2010).

Significant variation in grain $\mathrm{Zn}$ concentration can be found among different rice genotypes. The concentration of $\mathrm{Zn}$ ranged between 15.9 and $58.4 \mathrm{mg} \mathrm{kg}^{-1}$ for 939 brown rice samples evaluated at IRRI (Graham et al., 1999) and from 13.32 to $43.65 \mathrm{mg} \mathrm{Zn}$ $\mathrm{kg}^{-1}$ in 274 samples from China's germplasm (Jiang et al., 2008). Since the target $\mathrm{Zn}$ concentration for biofortification strategies is $28 \mu \mathrm{g} \mathrm{g}^{-1}$ dry weight for rice grains it has been suggested that this might be achieved through plant breeding (Bouis and Welch, 2010; White and Broadley, 2011).

Studies have reported variation in $\mathrm{Zn}$ concentrations in different tissues. For instance, the highest $\mathrm{Zn}$ concentration (179 $\mathrm{mg} \mathrm{kg}^{-1}$ ) 
was found in the embryo, followed by the aleurone $\left(51 \mathrm{mg} \mathrm{kg}^{-1}\right.$ ) and the endosperm (21 mg kg${ }^{-1}$ ) (Saenchai et al., 2012). The variation in $\mathrm{Zn}$ concentration among different grain tissues affects the concentration in the whole kernel in rice (Hansen et al., 2009; Saenchai et al., 2012) in a manner similar to wheat (Cakmak et al., 2010). Therefore, a technique that takes into account the variation among the tissues and their contribution to whole grain $\mathrm{Zn}$ concentration should be useful in germplasm evaluation and breeding. However, milling chemical analysis are high cost per samples and request expensive infrastructure (Pfeiffer and McClafferty, 2007). Staining with dithizone (DTZ) is an inexpensive technique to detect $\mathrm{Zn}$ in tissues (Ozturk et al., 2006; Velu et al., 2008; Shobhana et al., 2013). Dithizone is a Zn-chelating agent that binds $\mathrm{Zn}$ complex through an intense red color produced by $\mathrm{Zn}$ dithizone complex (Cakmak et al., 2010; Ozturk et al., 2006). Dithizone stains has also been used to localize $\mathrm{Zn}$ grain (Jaksomsak et al., 2014; Prom-u-thai et al., 2010). However, these previous studies did not quantify the content of $\mathrm{Zn}$ in each tissue to the total grain $\mathrm{Zn}$ concentration. This study aimed to: (i) to estimate the intensity of DTZ staining in brown rice grain tissues using Image software, (ii) to estimate the accuricity of DTZ staining and correlation with total $\mathrm{Zn}$ in grains, and (iii) to evaluate genotypic variation in $\mathrm{Zn}$ concentration of rice grain using chemical and DTZ staining.

\section{Materials and methods}

\subsection{Plant material and sample preparation}

Seeds of six upland rice (Oryza sativa L.) accessions (Table 1) were provided by Upland Rice Breeding Program, which is developed in Minas Gerais state (Brazil); EPAMIG (Agriculture and Livestock Research Institute), UFLA (Federal University of Lavras), and EMBRAPA (Brazilian Agricultural Research Corporation). A field experiment was undertaken in 2011/2012 in a paddy field at Value for Cultivation and Use (VCU) in the EPAMIG Experimental Station (EELA), in Lavras, MG, Brazil (latitude: $21^{\circ} 14^{\prime} \mathrm{S}$; longitude: $45^{\circ} 00^{\prime} \mathrm{W}$ and elevation: 918.841 above msl) during the rice growing season. VCUs are made for a particular regional scope, or for one or more Brazilian states and have usually been applied in plant research for annual plants to select the best accessions based on genotypic value for cultivar recommendation. Rice plants were grown in sandy clay soil under upland conditions with additional irrigation at EELA. The experimental design was a randomized complete block with three replications. Each plot consisted of five rows of $5.0 \mathrm{~m}$, spaced $0.4 \mathrm{~m}$ apart and a density of 80 seeds per metre. Basal fertilization consisted of the mixture of $400 \mathrm{~kg} \mathrm{ha}^{-1}$ of NPK fertilizer (08-28$16)+0.5 \% \mathrm{Zn}$ and top dressing with $100 \mathrm{~kg} \mathrm{ha}^{-1}$ of $\mathrm{N}$ (ammonium sulfate) was applied in two equal portions ( 25 and 45 days from sowing) (typical fertilization for trials testing the Value for Cultivation and Use - VCU). Rice grains were harvested at full maturity for $\mathrm{Zn}$ analyses.

\subsection{Chemical analysis}

Rice grains from ten plants per plot were harvested and ovendried at $65{ }^{\circ} \mathrm{C}$ until the grain reached a constant weight prior to analysis. Grain was dehusked in a testing husker (model MT, SUZUKI) to obtain brown rice caryopses (unpolished). For $\mathrm{Zn}$ analysis, $100 \mathrm{mg}$ samples of brown rice caryopses were digested using $4 \mathrm{~mL}$ of concentrated $\mathrm{HNO}_{3}$ and $2 \mathrm{~mL}$ of concentrated $\mathrm{HClO}_{4}$ (Sigma-Aldrich, Saint Louis, MO, USA) at $120^{\circ} \mathrm{C}$ for $1 \mathrm{~h}$ and then at $220^{\circ} \mathrm{C}$ until $\mathrm{HClO}_{4}$ fumes were observed. Total $\mathrm{Zn}$ concentrations in the samples were determined by atomic absorption spectrophotometry (AAS, PerkinElmer Inc., San Jose, CA, USA) (Malavolta et al., 1997) with three replications. Tomato leaf (SRM 1573A) and rice flour (SRM 1568A) standards (National Institute of Standards and Technology, Gaithersburg, MD) were digested and analyzed along side the rice samples to ensure accurate and reliable analytical data.

\subsection{Diphenylthiocarbazone (dithizone) staining}

About twenty rice grains were dehusked manually, excised longitudinally along the crease by a scalpel, and then submerged in freshly-prepared DTZ solution, by dissolving 1,5-diphenyl thiocarbazone (Merck) (500 $\mathrm{mg} \mathrm{L}^{-1}$ ) in methanol (reagent grade) for $30 \mathrm{~min}$, as described previously (Ozturk et al., 2006). Samples were rinsed thoroughly in distilled deionized water and blotted dry using tissue paper.

\subsection{Image acquisition and processing}

Rice grains stained using DTZ were photographed at a $6 \times$ magnification using a stereoscopic zoom microscope (Nikon SMZ 1500, Japan) and a Camera Control Unit (Nikon, SU-1). The 24 bitdepth images were analyzed on a desktop or laptop computer using ImageJ software (Ferreira and Rasband, 2012). The intensity of staining was measured through the RGB color space (Red, Green and Blue) defined by formula staining intensity values expressed as $R+G+B / 3$. Intensity data represented the relative density of $\mathrm{Zn}$ in the grains, and was scored from 1 (less intense color), 2 (medium intense color), 3 (intense color) to 4 (very intense color) in accordance with the intensity of staining (RGB values, scale from 0 to 255).

\subsection{Staining intensity analysis}

Staining intensity values $(R+G+B / 3)$ from RGB images were evaluated by ImageJ software and then scored as $1,2,3$ or 4 , according to frequency distribution in 4-class interval as following in Eq. (1):

Table 1

Zinc concentration in grains and information about 6 upland rice accessions used in our study.

\begin{tabular}{|c|c|c|c|}
\hline Identification & $\mathrm{Zn}\left(\mathrm{mg} \mathrm{kg}^{-1}\right)^{\mathrm{a}}$ & Accessions (release year) ${ }^{\mathrm{b}}$ & Recommendations $^{c}$ (Brazilian states) \\
\hline 1 & $29.6 \pm 0.59$ & BRS Esmeralda (2012) & MT, GO, MA, MG, PA, PI, RO, RR, TO \\
\hline 2 & $31.6 \pm 1.27$ & Line MG 10976 & - \\
\hline 3 & $32.3 \pm 1.07$ & BRSMG Relâmpago (2007) & MG \\
\hline 4 & $27.2 \pm 0.55$ & BRSMG Caravera (2007) & MG \\
\hline 5 & $36.5 \pm 1.14$ & Line CMG 1510 & - \\
\hline 6 & $30.1 \pm 0.93$ & BRSMG Curinga (2004) & MG, GO, MT, MS, TO, AM, RO, MA, PA, PI \\
\hline
\end{tabular}

a Zinc concentration in brown rice (husks removed) were determined by atomic absorption spectrophotometry (AAS).

b Accessions included 4 cultivars and 2 lines of upland rice.

c Refers to some locations of the regions where the cultivars or lines are tested and then suggested for growing. 
score $1=\left[\operatorname{Min}(R+G+B / 3)-(R+G+B / 3)_{1}>\right.$;

score $2=\left[(R+G+B / 3)_{1}-(R+G+B / 3)_{2}>\right.$;

score $3=\left[(R+G+B / 3)_{2}-(R+G+B / 3)_{3}>\right.$;

score $4=\left[(R+G+B / 3)_{3}-\operatorname{Max}(R+G+B / 3)>\right.$;

Where " $1>$ " mean the superior limit of the first class interval (score 1) as well as " $2>$ " mean the superior limit of the second class interval (score 2), etc. Moreover, $\operatorname{Min}(R+G+B / 3)$ and $\operatorname{Max}(R+G+B / 3)$ represents the lowest and highest staining intensity values $(R+G+B / 3)$, respectively, measured by Image J software analysis.

The Eq. (2) below was adapted according to the equation used by Pintasen et al. (2007) to calculate the staining intensity index among different seed lots of upland rice germplasm. Therefore, to calculate the staining intensity index $(Y)$ within each region (embryo, endosperm or aleurone) of the rice seeds, Eq. (2) was used: ranged from 27.2 to $36.5 \mathrm{mg} \mathrm{kg}^{-1}$ (Table 1 ). Considering $\mathrm{Zn}$ concentration in brown rice, which is the edible portion after dehusking process, the highest $\mathrm{Zn}$ concentration was found in accession 5 (36.5 $\mathrm{mg} \mathrm{kg}^{-1}$ ), whereas accession 4 had the lowest $\left(27.2 \mathrm{mg} \mathrm{kg}^{-1}\right.$ ). Moreover, the DTZ staining showed comparable intensity of $\mathrm{Zn}$ localization when viewing the whole intact kernels, but the staining intensity of each grain region was more clearly differentiated in the longitudinal sections of the kernels, which was chosen for staining intensity analysis of rice grain among different regions and accessions through ImageJ software (Supplementary Fig. 1).

\subsection{Staining intensity through image analysis}

The images of each stained section were rescored using the staining intensity values $(R+G+B / 3)$. The Image J software was used to analyze the $R+G+B / 3$ values from RGB images in different

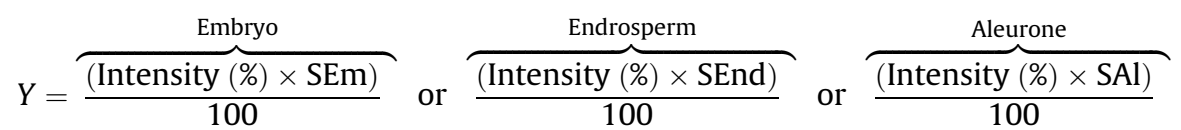

regions of rice seed studied (embryo, endosperm and aleurone zones) (Fig. 1A). There were different levels of staining intensity (RGB intensity $\times$ distance) among regions of the seeds such as embryo (Fig. 1B), aleurone layer (Fig. 1C) and endosperm (Fig. 1D), yet they were in concordance with the sensitivity of red tones perceptible to the human eye.

The $R+G+B / 3$ values from RGB images were used to calculate the staining intensity index $(Y)$ as shown in Eq. (2). The significant variation of the index was found among seed regions (the embryo, endosperm, and aleurone) of six rice accessions (Fig. 2). Half of the analyzed rice accessions (numbers 1,3 , and 4 ), the higher index $(p<0.05)$ was found in the embryo compared to the aleurone and endosperm regions. On these three accessions, the index follows the order embryo $>$ aleurone $>$ endosperm. However, the highest index for the endosperm and aleurone regions was found for the accession 5. There was no difference of the index among the 3 regions in accession 6 .

The area of each stained region was different within and between accessions (Fig. 3A). The stained area of the endosperm was about 10 times larger than of the embryo and aleurone regions in all accessions. The index weighted by stained area of each region $(Y A W)$ was used to represent the staining intensity of each region in the whole kernel.

Combining the $Y A W$ for the 3 regions gave whole grain $Y A W$ that indicated significant variation in the amount of $\mathrm{Zn}$ in the grain among the 6 accessions studied ( $\mathrm{p}<0.05)$ (Fig. 3B). Accession 5 had the highest whole grain YAW and the lowest was found in accessions 1, 2, 4, and 6. The total YAW among 6 accession was positively correlated with $\mathrm{Zn}$ concentration in brown rice kernel by chemical analysis $\left(r^{2}=0.74, p<0.05\right)$ (Fig. 4).

\section{Discussion}

\subsection{Concentration and localization of zinc in rice grains}

The chemical analysis showed a variation of $\mathrm{Zn}$ concentration in the kernel of 6 rice accessions studied, which was reported previously in several germplasms (Graham et al., 1999; Saenchai et al.,
A genotypic variation in $\mathrm{Zn}$ concentration was found in six Brazilian upland rice accessions for brown rice (husks removed), which

\section{(2)}




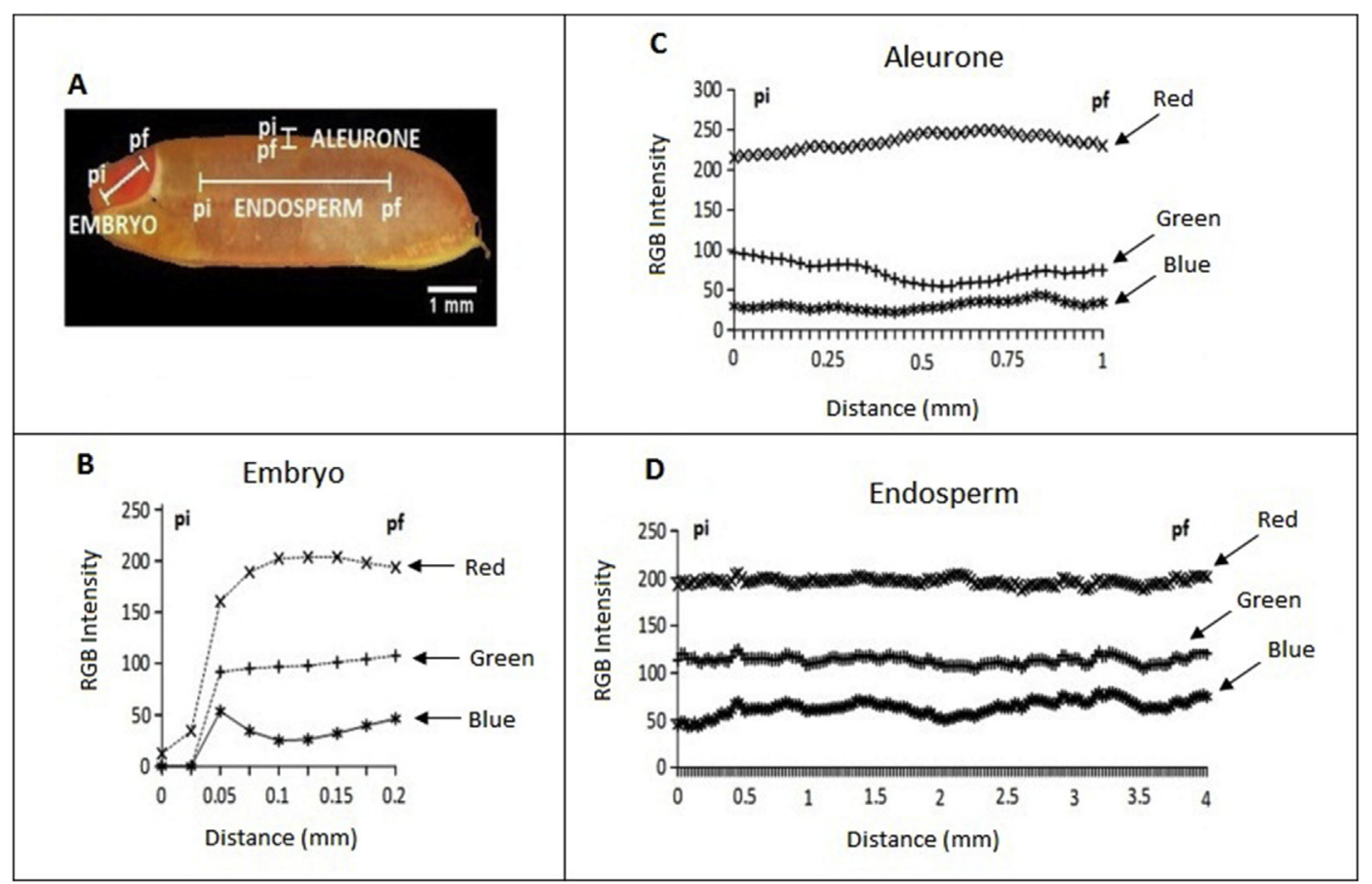

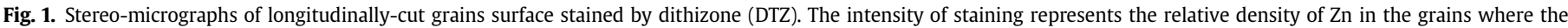

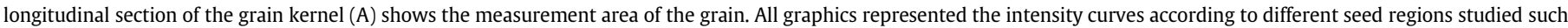
as embryo (B), aleurone (C) and endosperm (D) by ImageJ software. Distance on the $x$-axis shows the length of the studied grain regions. Points: initial (pi) and final (pf).

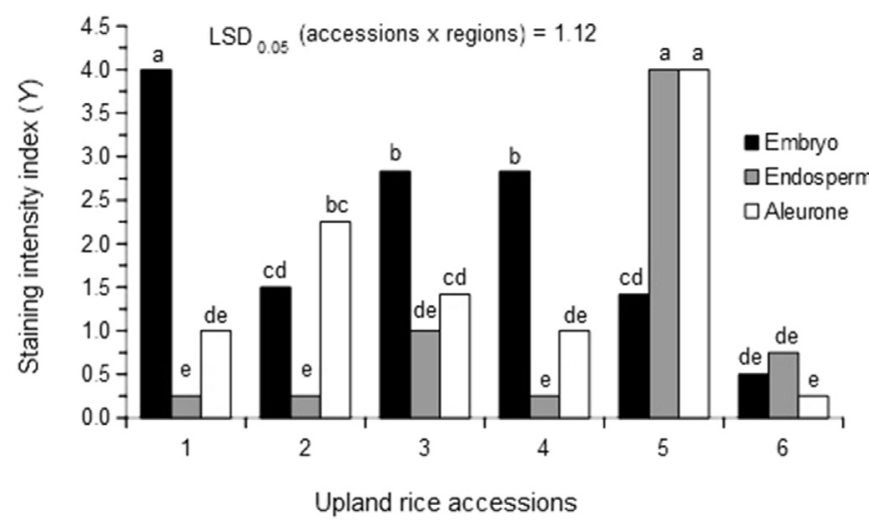

Fig. 2. The staining intensity index $(Y)$ among grain regions of 6 upland rice accessions from Brazil. Values shown represent means (bars) calculated from three independent replications.

2012). The variation of $\mathrm{Zn}$ in grains among rice accessions probably depends on the ability of $\mathrm{Zn}$ to mobilize from one part of the plant to the others such as from the husk to the grain. Recently, Yoneyama et al. (2010) reported that $\mathrm{Zn}$ redistribution for the grains and husks might be via the phloem after mobilization from the flag leaf and leaves below the flag leaf. However, several mechanisms may affect Zn remobilization such as the plant's nitrogen nutrition, the micronutrient transporters genes (e.g., ZIP family), the $\mathrm{pH}$ of the phloem sap and importantly, the forms of $\mathrm{Zn}$ chelators as nicotianamine (NA) and deoxymugineic acid (DMA) in the plant (Bashir et al., 2012; Cakmak et al., 2010; Clemens et al., 2013). Mediated by nicotianamine synthase (NAS) genes, NA plays an important role in the intercellular and long-distance transport of $\mathrm{Zn}$ and has also contributed to $\mathrm{Zn}$ mobilization into the endosperm (Clemens et al., 2013; Takahashi et al., 2009). However, it has also been indicated that $\mathrm{Zn}$ concentration in the grain was affected by the proportion of $\mathrm{Zn}$ in different grain regions both in rice (Hansen et al., 2009; Saenchai et al., 2012) and wheat (Cakmak et al., 2010). Thus, the appropriate localization of $\mathrm{Zn}$ intensities in different parts of a seed is a key factor for $\mathrm{Zn}$ concentration in the whole kernel of rice. In our study, the staining of longitudinal grain section with DTZ indicated that $\mathrm{Zn}$ is mostly localized in the embryo and aleurone layer parts, which agrees with previous investigations in several kinds of cereal grains by using the similar staining method (Cakmak et al., 2010; Ozturk et al., 2006; Shobhana et al., 2013; Velu et al., 2008). On the other hand, when the staining intensity is observed in tissues of rice grains by human eyes, it can be difficult to distinguish, especially when dealing the narrow variation among grain tissues. Therefore, the staining intensity carried through DTZ method and being visualized by image software may solve part of these problems aforementioned. In that perspective, we suggest this technique as an important tool for studies that involve the localization of $\mathrm{Zn}$ in different regions of rice grains.

\subsection{Assessing staining intensity through imageJ analysis}

Several approaches have been developed to investigate nutritional features in grains of cereal crops by using the staining technique (Ozturk et al., 2006; Jaksomsak et al., 2014; Prom-u-thai et al., 2010; Velu et al., 2008; Shobhana et al., 2013), which generate images. However, to our knowledge, the use of ImageJ software for specific analysis and measurement of stained grains is still rare. The measurement of staining intensity $(I)$ by ImageJ software analysis as demonstrated by profile of intensity was calculated to the staining intensity index $(Y)$ for the comparable of the intensity level as investigated in the previous studies (1: less intense; 2 : medium intense; 3: intense; 4: very intense) (Jaksomsak et al., 2014; Pintasen et al., 2007). When we used these indices, our results of rice staining showed that on embryo surface of analyzed grains had the highest $\mathrm{Zn}$ concentration compared with the endosperm and 


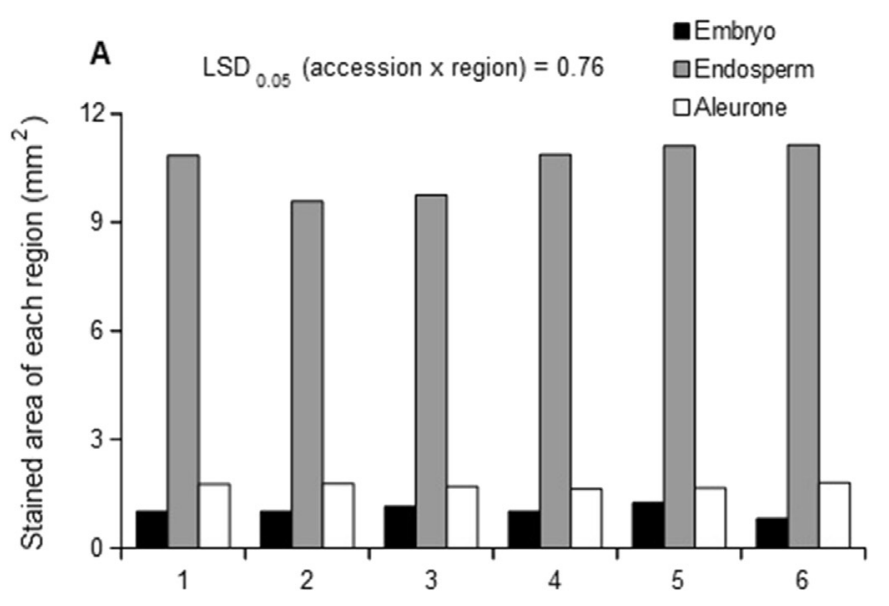

B

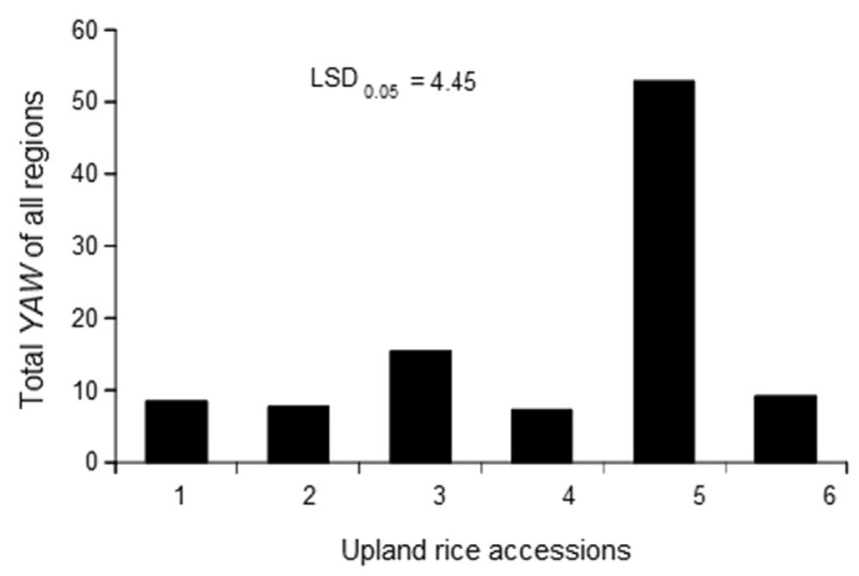

Fig. 3. The stained area of each grain regions (A) and total $Y A W$ of rice kernel (B) from 6 upland rice accessions. Total YAW is the sum of the staining intensity index weighted by stained area of each region $(Y A W)$ in the embryo, aleurone layer and endosperm as in the following Eq. (3): Total YAW $=\Sigma[(Y$ embryo $\times$ area $)+(Y$ aleurone $\times$ area $)+(Y$ endosperm $\times$ area)]. For stained area, values shown represent means (bars) calculated from three independent replications.

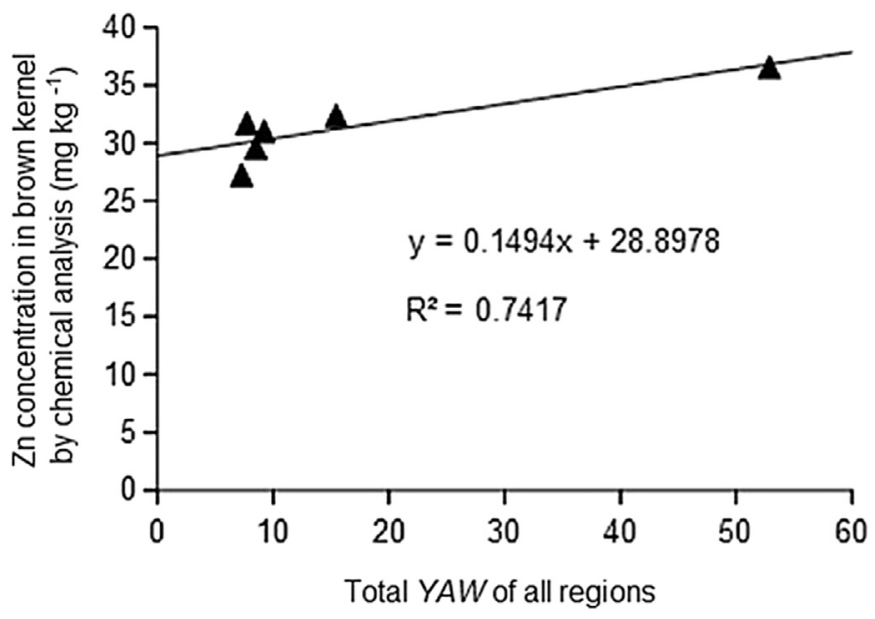

Fig. 4. Relationship between $\mathrm{Zn}$ concentration in brown rice from chemical analysis and total YAW of all regions from 6 upland rice accessions. Total YAW is the sum of the staining intensity index weighted by stained area of each region (YAW) in the embryo, aleurone layer and endosperm as in the following equation: Total $Y A W=\Sigma[(Y$ embryo $\times$ area $)+(Y$ aleurone $\times$ area $)+(Y$ endosperm $\times$ area $)]$. aleurone layer in most accessions. This is consistent with recent findings of advanced method, which revealed a similar distribution pattern of $\mathrm{Zn}$ in the embryo of rice grains by Synchrotron X-ray fluorescence microscopy (XFM) (Lu et al., 2013; Kyriacou et al., 2014; Takahashi et al., 2009). However, the XFM technique may not be as easily available or accessible compared to the staining method addressed in this essay together with the Image software. Previous studies reported that the distribution of $\mathrm{Zn}$ was considerably localized in the embryo and aleurone layer of wheat (Ozturk et al., 2006) and rice kernels (Lu et al., 2013). However, the higher $Y A W$ index found in the endosperm of the grain compared with the other regions was probably due to the much larger endosperm surface area in those grains (Fig. 3A), demonstrated by the images from our analysis. The higher $\mathrm{Y}$ in the aleurone and endosperm than in the embryo of accession 5 contributed the large portion to YAW could be due to cross contamination from other regions of embryo and aleurone layer which can be occurred during soaking and sectioning of rice grain before the staining procedures. The contamination of $\mathrm{Zn}$ from other region to the endosperm resulted in high staining intensity value $R+G+B / 3$ which was used to calculate $\mathrm{Y}$. This cause of the higher endosperm $\mathrm{Y}$, rather than a larger endosperm surface area, that contributed to a higher $Y A W$ for accession 5 than others. Therefore, attention should be made when preparing the grain samples for $\mathrm{Zn}$ staining to avoid contamination between the regions of rice grain and/or other sources e.g., lazor blade and laboratory glass wares.

\subsection{Correlation analysis}

The significant variation of $\mathrm{Zn}$ concentration in brown rice kernel was complemented by measuring the staining intensity weight by stained area of the embryo, endosperm, and aleurone regions. The staining intensity method showed that various regions of the grain contained differentiated amounts of $\mathrm{Zn}$ concentration. The contribution was greater from the endosperm and aleurone regions due to their larger area compared with embryo in the image analysis. The total $\mathrm{Zn}$ concentration in the whole caryopsis is contributed by the staining intensity $\mathrm{Zn}$ in all regions as it was observed from the significant relationship between total $\mathrm{Zn}$ concentration in the caryopsis and the total YAW $\left(\mathrm{r}^{2}=0.7417, p<0.05\right)$ (Fig. 4). If the total YAW is established from the staining intensity of DTZ, for example total $Y A W=30$, the concentration of $\mathrm{Zn}$ in brown rice caryopsis would be estimated as $33.38 \mathrm{mg} \mathrm{kg}^{-1}$ which could be valuable and convenient in the conventional laboratory where atomic absorption spectrophotometry is not existed. However, there was a weak point that could be observed in this method. For example, a wide gap between the total YAW and grain $\mathrm{Zn}$ concentration of both brown rice caryopsis and rough rice of accession 5 was observed and could be explained by the sensitivity of the staining intensity and YAW calculations. As described above that $Y A W$ is the index use to represent the staining intensity of each region in the whole kernel calculated by multiplying the staining intensity index $(Y)$ within each stained region determined by the imageJ software with their area. Therefore, the indication of the staining intensity and the stained region is highly sensitive to the total YAW. For example, if there is any contamination of $\mathrm{Zn}$ staining across the regions and over calculation from the stained regions would make high Y value and consequently resulted in high total YAW as appeared in accession 5. Otherwise, our methodology indicates that manipulating the ImageJ software together with the DTZ staining can be efficiently used to assess the variation of $\mathrm{Zn}$ concentration between rice accessions and within the grain tissues from seeds, e.g., the endosperm and aleurone layer. It is a rapid, easy and economical method to evaluate $\mathrm{Zn}$ concentration in rice grain, especially when dealing with large germplasms (Shobhana 
et al., 2013; Velu et al., 2008). This method allows plant breeders to determine the relative concentrations of $\mathrm{Zn}$ in rice within and between seed lot when working with rice samples that are genetically diverse (Pintasen et al., 2007; Prom-u-thai et al., 2003). However, thorough and specific evaluations of the small regions such as the embryo or the scutellum would be necessary to improve this method for further research and development.

\section{Conclusions}

This study confirms that the variation of $Z n$ concentration can be distinguished among rice accessions, seed lots, and grain tissues by using DTZ staining together with the Image software analysis. The total staining intensity multiply by the stained regions (YAW) can be used to estimate grain $\mathrm{Zn}$ concentration, which can be convenient among the conventional laboratory without Atomic Absorption Spectrophotometer. The staining method is easy, rapid, and economically viable and Image software is freely accessible imaging program. This is an important finding for rice breeders interested in developing high-density $\mathrm{Zn}$ genotypes to solve the problem of $\mathrm{Zn}$ deficiency among the world's population. The screening procedure may be carried out even in large number of samples without the problem of small amount per sample of rice, a problem that often occurs when performing chemical analysis. Even though, cross-sectioning is still a manual rather than an automated operation which would limit the number of samples that can be analyzed, further research requiring development of the specific tools and further refined by calibrating the staining surface to identify more closely the contribution of different tissues to total grain $\mathrm{Zn}$.

\section{Acknowledgments}

The authors are grateful to the Brazilian agencies CNPq, CAPES and FAPEMIG and National Research University Programme of Thailand's Commission on Higher Education for financial support. The authors are also grateful to the Laboratory of Electron Microscopy and Ultrastructural Analysis (LME/UFLA), AgroMetals Research Network and Prof. Benjavan Rerkasem for her valuable comments on the manuscript.

\section{Appendix A. Supplementary data}

Supplementary data related to this article can be found at http:// dx.doi.org/10.1016/j.jcs.2015.11.006.

\section{References}

Alloway, B.J., 2008. Zinc in Soils and Crop Nutrition, second ed. IZA and IFA Brussels, Belgium and Paris, France.

Bashir, K., Ishimaru, Y., Nishizawa, N.K., 2012. Molecular mechanisms of zinc uptake and translocation in rice. Plant Soil 361, 189-201.

Bouis, H.E., Welch, R.M., 2010. Biofortification - a sustainable agricultural strategy for reducing micronutrient malnutrition in the global south. Crop Sci. 50, S20-S32.

Cakmak, I., 2008. Enrichment of cereal grains with zinc: agronomic or genetic biofortification? Plant Soil 302,1-17.
Cakmak, I., Pfeiffer, W.H., McClafferty, B., 2010. Biofortification of durum wheat with zinc and iron. Cereal Chem. 87, 10-20.

Clemens, S., Deinlein, U., Ahmadi, H., Höreth, S., Uraguchi, S., 2013. Nicotianamine is a major player in plant $\mathrm{Zn}$ homeostasis. Biometals 26, 623-632.

Ferreira, T., Rasband, W., 2012. ImageJ User Guide IJ 1.46r. http://rsb.info.nih.gov/ij/ docs/guide/user-guide.pdf (accessed November, 2013).

Graham, R., Senadhira, D., Beebe, S., Iglesias, C., Monasterio, I., 1999. Breeding for micronutrient density in edible portions of staple food crops: conventional approaches. Field Crops Res. 60, 57-80.

Graham, R.D., Knez, M., Welch, R.M., 2012. How much nutritional iron deficiency in humans globally is due to an underlying zinc deficiency? Adv. Agron. 115, 1-40.

Hansen, T.H., Laursen, K.H., Persson, D.P., Pedas, P., Husted, S., Schjoerring, J.K., 2009 Micro-scaled high-throughput digestion of plant tissue samples for multielemental analysis. Plant Methods 5, 12.

Jaksomsak, P., Sangruan, P., Thomson, G., Rerkasem, B., Dell, B., Prom-u-Thai, C., 2014. Uneven distribution of zinc in the dorsal and ventral sections of rice grain. Cereal Chem. 91, 124-129.

Jiang, S.L., Wu, J.G., Thang, N.B., Feng, Y., Yang, X.E., Shi, C.H., 2008. Genotypic variation of mineral elements contents in rice (Oryza sativa L.). Eur. Food Res. Technol. 228, 115-122.

Kyriacou, B., Moore, K.L., Paterson, D., Jonge, M.D., Howard, D.L., Stangoulis, J., Tester, M., Lombi, E., Johnson, A.A.T., 2014. Localization of iron in rice grain using synchrotron X-rayfluorescence microscopy and high resolution secondary ion mass spectrometry. J. Cereal Sci. 59, 173-180.

Lu, L., Tian, S., Liao, H., Zhang, J., Yang, X., Labavitch, J.M., Chen, W., 2013. Analysis of metal element distributions in rice (Oryza sativa L.) seeds and relocation during germination based on X-Ray fluorescence imaging of Zn, Fe, K, Ca, and Mn. PLoS ONE 8, 1-9.

Malavolta, E., Vitti, G.C., Oliveira, S.A., 1997. Avaliação do estado nutricional das plantas: princípios e aplicações, second ed. Potafos, Piracicaba, SP, Brazil (in Portuguese).

Ozturk, L., Yazici, M.A., Yucel, C., Torun, A., Cekic, C., Bagci, A., Ozkan, H., Braun, H.J. Sayers, Z., Cakmak, I., 2006. Concentration and localization of zinc during seed development and germination in wheat. Physiol. Plant. 128, 144-152.

Pfeiffer, W.H., McClafferty, B., 2007. Harvestplus: breedings crops for better nutrition. Crop Sci. 47, S88-S105.

Pintasen, S., Prom-u-thai, C., Jamjod, S., Yimyam, N., Rerkasem, B., 2007. Variation of grain iron content in a local upland rice germplasm from the village of Huai Tee Cha in northern Thailand. Euphytica 158, 27-34.

Prasad, A.S., 2012. Discovery of human zinc deficiency: 50 years later. J. Trace Elem. Med. Biol. 26, 66-69.

Prom-u-thai, C., Dell, B., Thomson, G., Rerkasem, B., 2003. Easy and rapid detection of iron in rice grain. Sci. Asia 29, 213-217.

Prom-u-thai, C., Rerkasem, B., Cakmak, I., Huang, L., 2010. Zinc fortification of whole rice grain through parboiling process. Food Chem. 120, 858-863.

Saenchai, C., Prom-u-thai, C., Jamjod, S., Dell, B., Rerkasem, B., 2012. Genotypic variation in milling depression of iron and zinc concentration in rice grain. Plan Soil 361, 271-278.

Sharma, A., Patni, B., Shankhdhar, D., Shankhdhar, S.C., 2013. Zinc - an indispensable micronutrient. Physiol. Mol. Biol. Plants 19, 11-20.

Shobhana, V.G., Senthil, N., Kalpana, K., Abirami, B., Sangeetha, J., Saranya, B., Jana Jeevan, R., Tamilkumar, P., Nagarajan, P., Velu, V., Arumugachamy, S., John Joel, A., Vellaikumar, S., Raveendran, M., 2013. Comparative studies on the iron and zinc contents estimation using atomic absorption spectrophotometer and grain staining techniques (Prussian Blue and DTZ) in maize germplasms. J. Plant Nutr. 36, 329-342.

Takahashi, M., Nozoye, T., Kitajima, N., Fukuda, N., Hokura, A., Terada, Y., Nakai, I., Ishimaru, Y., Kobayashi, T., Nakanishi, H., Nishizawa, N.K., 2009. In vivo analysis of metal distribution and expression of metal transporters in rice seed during germination process by microarray and X-ray fluorescence imaging of Fe, Zn, Mn, and Cu. Plant Soil 325, 39-51.

Velu, G., Bhattacharjee, R., Rai, K.N., Sahrawat, K.L., Longvah, T., 2008. A simple and rapid screening protocol for grain Zn content in pearl millet. J. SAT Agric. Res. 6, $1-4$.

White, P.J., Broadley, M.R., 2009. Biofortification of crops with seven mineral elements often lacking in human diets - iron, zinc, copper, calcium, magnesium, selenium and iodine. New Phytol. 182, 49-84.

White, P.J., Broadley, M.R., 2011. Physiological limits to zinc biofortification of edible crops. Front. Plant Sci. 2, 1-11.

Yoneyama, T., Gosho, T., Kato, M., Goto, S., Hayashi, H., 2010. Xylem and phloem transport of $\mathrm{Cd}, \mathrm{Zn}$ and $\mathrm{Fe}$ into the grains of rice plants (Oryza sativa L.) grown in continuously flooded Cd-contaminated soil. J. Soil Sci. Plant Nutr. 56, 445-453. 